\title{
Pulmonary auscultation terminology employed in Brazilian medical journals between January of 1980 and December of 2003*
}

\author{
KAMILA FERNANDA STASZKO', CARLA LINCHO', VIVIAN DA CAS ENGELKE', NÁDIA SPADA FIORI', \\ KARINA CIRINO SILVA', ELISA IRIBARREN NUNES', LINJIE ZHANG ${ }^{2}$
}

\begin{abstract}
Objective: To evaluate the appropriateness of the use of auscultation terminology in Brazilian respiratory diseaserelated medical journals published between January of 1980 and December of 2003. Methods: A descriptive study was conducted, evaluating three medical journals: the Jornal de Pneumologia (Journal of Pulmonology), Jornal de Pediatria (Journal of Pediatrics) and Revista Brasileira de Medicina (Brazilian Journal of Medicine). Original articles and case reports about respiratory diseases were selected, and auscultation terminology was extracted from these articles. The appropriateness of terms used to describe adventitious sounds was assessed. Results: We found that the inappropriate use of terms was more frequent when intermittent sounds were described than when continuous sounds were described ( $87.7 \%$ versus $44.0 \% ; p=0.0000$ ). No significant difference was observed between the inappropriate use of terms by pulmonologists and that observed for other specialists ( $56.5 \%$ versus $62.0 \% ; p=0.26)$. In addition, there were no significant differences among the various regions of the country or between the periods prior to and after the dissemination of international nomenclature. Conclusion: Inappropriate use of pulmonary auscultation terms describing adventitious sounds remains common and widespread in Brazilian medical publications.
\end{abstract}

Keywords: Auscultation; Lung/physiopathology; Lung diseases/diagnosis; Respiratory sounds; Terminology

\footnotetext{
* Study carried out at the Fundação Universidade Federal do Rio Grande (FURG, Federal University of Rio Grande Foundation), Rio Grande do Sul, Brazil.

1. Student of Medicine at the Federal University of Rio Grande, Rio Grande do Sul, Brazil.

2. Assistant Professor of the Maternal and Infant Department of the Federal University of Rio Grande, Rio Grande do Sul, Brazil.

Correspondence to: Linjie Zhang. Rua Visconde Paranaguá s/n, Centro - CEP: 96201-000, Rio Grande do Sul (RS) Brasil.

Tel: 5553 3233-9945. E-mail: zhanglinjie63@yahoo.com.br

Submitted: 10 August 2005. Accepted, after review: 9 February 2006.
} 
Terminologia da ausculta pulmonar utilizada em publicações médicas brasileiras,

\section{INTRODUCTION}

The importance of respiratory auscultation has been made evident since the invention of the stethoscope by Laennec, which allowed a more accurate diagnosis of pleuropulmonary diseases. ${ }^{(1)}$ Even after the great technological advances in clinical diagnosis, respiratory auscultation remains a useful tool for evaluating patients with respiratory diseases. However, there is still much confusion regarding the terminology used to describe pulmonary auscultation. ${ }^{(2-5)}$ This motivated specialists from several countries to meet in 1985 in order to standardize the nomenclature, aiming to simplify it, as well as to include new concepts in pulmonary auscultation. ${ }^{(6)}$ Since that time, new pulmonary auscultation terms have become prevalent in the international literature. ${ }^{(7-9)}$

In Brazil, the impact that these terminological modifications have had on medical practice remains unknown. A recent study demonstrated that these terminological modifications have not been incorporated into practice by medical residents and interns at a university hospital. ${ }^{(10)}$ Another study concluded that even pulmonologists were unfamiliar with the current nomenclature. ${ }^{(11)}$ Of the 131 case reports analyzed in that study, 72 used incorrect terms to describe pulmonary auscultation, employing 30 different designations to describe breath sounds. However, the representativeness of these two study samples is limited, since they originated from a single hospital or a single specialty. In addition, the evolution of the appropriateness of the use of pulmonary auscultation terms has not been studied since the dissemination of the new international nomenclature.

This descriptive study aimed to evaluate the appropriateness of the use of pulmonary auscultation terms in articles about respiratory diseases published in Brazilian medical journals.

\section{METHODS}

A descriptive study was carried out based on data extracted from some Brazilian medical journals between January of 1980 and December of 2003. Three medical journals were selected, each from a distinct area of study - pulmonology, pediatrics, and clinical practice - according to the following criteria: indexed for MEDLINE, for the Index Medicus Latino Americano (IMLA, Latin American Index Medicus) database, or for the Literatura Latinoamericana y del Caribe en Ciencias de la Salud (Latin American and Caribbean Health Sciences Literature, sucessor to the IMLA) database; and available in the library of the Federal University of Rio Grande.

Data were collected in two steps. First, six fifthyear medical students were divided into three groups of two and were asked to select the potential articles for the study. Each group was responsible for one journal. Each examiner independently analyzed the title and the abstract of all published articles in order to select the potential articles, that is, the respiratory disease-related articles. In this step, the total number of articles, as well as the number of original articles and case reports, was recorded. After the selection process, the two examiners in each group compared their results, and differences were resolved by consensus. In the second step, the two examiners in each group extracted data related to the pulmonary auscultation terms and other terms according to a predefined table. The search for data was focused on the "Methods" and "Results" sections of the articles selected. In this step, differences of opinion were also solved by reaching a consensus between the two examiners.

The terms used to describe adventitious sounds in pulmonary auscultation were classified as appropriate or inappropriate according to the terms established by the International Lung Sounds Association (ILSA) ${ }^{(6)}$ : continuous sounds (wheezing and rhonchi) and intermittent sounds (coarse and fine rales). The percentage of terms used appropriately was compared among the different regions of Brazil, and among the different medical specialties. Both of these determinations were based on the information provided for the first author of each article. Data were also compared between two periods (1980-1987 and 19882003), with the objective of evaluating the impact of the ISLA-approved nomenclature, disseminated in $1987,{ }^{(6)}$ on the use of pulmonary auscultation terms by Brazilian physicians.

Data from the extraction tables were digitalized, and the statistical analysis was carried out using the Statistics for Windows 4.3 program (Statsoft, Inc., 1993). The chi-square test was used to analyze categorical data. The $\alpha$-error was pre-established at 0.05 . 


\section{RESULTS}

Three journals were selected for this study: the Jornal de Pneumologia (Journal of Pulmonology, former name of the Jornal Brasileiro de Pneumologia, Brazilian Journal of Pulmonology); the Jornal de Pediatria (Journal of Pediatrics); and the Revista Brasileira de Medicina (Brazilian Journal of Medicine). A total of 2557 original articles and case reports were analyzed. Of those 2557 articles, respiratory disease-related articles accounted for $813(31.8 \%), 283(34.8 \%)$ of which described pulmonary auscultation terms.

Table 1 shows the description of continuous sounds in pulmonary auscultation. There were 6 different terms used, totaling 266 occurrences. Sibilos (wheezing) was the term most often used (in 36.1\% of the occurrences), followed by sibilância (sibilance, in 26.3\%), and roncos (rhonchi, in 19.2\%). As for the intermittent sounds, there were 154 occurrences of 20 different terms (Table 2). Estertores (rales) accounted for $20.7 \%$ of the occurrences, followed by estertores crepitantes (crackling rales, accounting for 18.8\%), and estertores subcrepitantes (subcrackling rales, accounting for $13.7 \%$ ).

Table 3 shows the inappropriateness of the terminology used to report the pulmonary auscultation, organized by the type of sound, the publication period, and the specialty of the author. A higher percentage of inappropriateness was observed for the terms used to describe intermittent sounds than for those used to describe continuous sounds $(87.7 \%$ vs. $44 \%$, $\mathrm{p}=0.0000$ ). There was no significant difference between the 1980-1987 period and the 1988-2003 period in the inappropriateness of the terms used to describe adventitious sounds ( $60.8 \%$ vs. $59.7 \%, p=$ 0.85 ). Nor were any significant differences observed

\section{TABLE 1}

Overview of the terms used to describe continuous sounds

\begin{tabular}{lcc}
\hline Continuous sounds & Number of occurrences & $\%$ \\
\hline Wheezing & 96 & 36.09 \\
Sibilance & 70 & 26.31 \\
Rhonchi & 51 & 19.17 \\
Wheeziness & 46 & 17.30 \\
Deep wheezing & 02 & 0.75 \\
Rhonchiness & 01 & 0.38 \\
\hline Total & 266 & 100 \\
\hline
\end{tabular}

TABLE 2

Overview of the terms used to describe intermittent sounds

\begin{tabular}{lrr}
\hline Intermittent sounds & Number of occurrences & \multicolumn{1}{c}{$\%$} \\
\hline Rales & 32 & 20.78 \\
Crackling rales & 29 & 18.83 \\
Subcrackling rales & 21 & 13.64 \\
Crackling & 18 & 11.69 \\
Bullous rales & 9 & 5.84 \\
Crackles & 2 & 1.30 \\
Cracklings & 6 & 3.89 \\
Small-bulla subcrackling rales & 1 & 0.65 \\
Medium-bulla subcrackling rales & 1 & 0.65 \\
Large-bulla subcrackling rales & 2 & 1.30 \\
Small-bulla rales & 3 & 1.94 \\
Medium-bulla rales & 4 & 2.60 \\
Large-bulla rales & 2 & 1.30 \\
Fine rales & 11 & 7.14 \\
Rhonchial rales & 1 & 0.65 \\
Ralings & 1 & 0.65 \\
Coarse rales & 7 & 4.55 \\
Wet rales & 1 & 0.65 \\
Fine crackling & 2 & 1.30 \\
Bullae & 1 & 0.65 \\
\hline Total & 154 & 100 \\
\hline
\end{tabular}

between pulmonologists and physicians engaged in other specialties ( $56.5 \%$ vs. $62 \%, p=0.26$ ).

Table 4 shows the inappropriateness of the terms by state. Due to the number of publications, the states were divided into three groups, by region: Southeast (São Paulo, Rio de Janeiro, and Minas Gerais); South (Paraná, Santa Catarina, and Rio

\section{TABLE 3}

Inappropriateness of the pulmonary auscultation terminology by type of sound, date of publication, and author specialty

\begin{tabular}{lccc}
\hline & $\begin{array}{c}\text { Total no. of } \\
\text { occurrences }\end{array}$ & $\begin{array}{c}\text { No. of } \\
\text { inapropriate } \\
(\%)\end{array}$ & $\mathrm{p}$ value \\
\hline $\begin{array}{l}\text { Sound type } \\
\text { Continuous }\end{array}$ & 266 & $117(44.0)$ & \\
$\begin{array}{l}\text { lntermittent } \\
\text { Publication period }\end{array}$ & 154 & $135(87.7)$ & $\mathrm{p}=0.0000$ \\
$\begin{array}{l}\text { 1980-1987 } \\
\text { 1988-2003 }\end{array}$ & 92 & $56(60.8)$ & \\
$\begin{array}{l}\text { Author specialty } \\
\text { Pulmonologist }\end{array}$ & 328 & $196(59.7)$ & $\mathrm{p}=0.85$ \\
$\quad$ Non-pulmonologist & 266 & $154(56.5)$ & \\
\hline
\end{tabular}




\section{TABLE 4}

Inappropriateness of the pulmonary auscultation terminology by geographic distribution of authors

\begin{tabular}{lcc}
\hline Region & $\begin{array}{c}\text { No. of occurrences } \\
\text { in articles }\end{array}$ & $\begin{array}{c}\text { No. of inappropriate } \\
\text { terms (\%) }\end{array}$ \\
\hline Southeast & 233 & $133(57.0)$ \\
South & 128 & $81(63.3)$ \\
Other & 59 & $38(64.4)$ \\
Total & 420 & $252(60.0)$ \\
\hline
\end{tabular}

$p=0.39$

Grande do Sul); and Other (Sergipe, Goiás, Mato Grosso, Mato Grosso do Sul, Bahia, Pernambuco, Ceará, Maranhão, and Amazonas). There were no significant differences among the regions in the terms used to describe adventitious sounds in pulmonary auscultation $(p=0.39)$.

\section{DISCUSSION}

Despite the importance of pulmonary auscultation for the diagnosis of respiratory diseases, this study showed that the correct terminology was used in only one-third of the respiratory disease-related articles. This indicates that medical researchers do not give this terminology the value it deserves.

The results of the present study show the high prevalence of inappropriate terms used to describe adventitious sounds, especially those related to intermittent sounds. Although the ILSA proposed only 2 terms to describe intermittent sounds, ${ }^{(6)} 20$ different terms were used. The wide range of terms used to describe intermittent sounds was also demonstrated in the two studies previously cited. ${ }^{(10-}$ 11) This wide range of descriptions, lacking clear definition criteria and well-defined bibliographical references, not only makes pulmonary auscultation subjective, but also impedes the teaching process and makes it difficult to draw comparisons between the semiological data extracted from scientific publications. According to the ILSA nomenclature, ${ }^{(6)}$ intermittent sounds are classified as fine and coarse rales. Fine crackles are produced by the serial aperture of previously closed airways. Fine crackles are generally associated with the presence of liquids or exudates in the alveoli, as seen in pneumonia, bronchiolitis, and left ventricular insufficiency. Fine crackles occur at the end of the inspiration, are acute (high frequency), are of short duration, and do not change during cough, although they do provoke a change in posture. Coarse crackles are created by the opening and closing of airways that contain dense, viscous secretion. The frequency of coarse crackles is lower (bass sounds), and they are of longer duration than are fine crackles. Coarse crackles are audible at the beginning of the inspiration and throughout the expiration, and they clearly change with cough. Coarse crackles are common in chronic bronchitis and bronchiectasis, in which secretion accumulates in the upper airways. These two terms define the acoustic characteristics of the sounds in a more objective way, which is important, since the presence of rales correlates well with pulmonary pathologies.

As for the continuous sounds, the situation is less alarming. Although the ILSA proposed only 2 terms to describe continuous sounds (wheezing and rhonchi), 6 different terms were used. Previous studies have obtained similar results. ${ }^{(10-11)}$ The fact that there was a greater degree of appropriateness among the terms used to describe continuous sounds than among those used to describe intermittent sounds might be related to the fact that continuous sounds are easier to distinguish. Rhonchi are deep sounds (of lower frequency) and wheezes are high sounds (of higher frequency).

Our findings show that the prevalence of inappropriateness in the description of adventitious sounds in the period after the dissemination of the new nomenclature was similar to that found for the period prior to their dissemination. This data indicates that, although the ILSA-approved nomenclature was disseminated 20 years ago, it has not yet been incorporated into Brazilian medical practice.

We observed no significant differences among specialties (of the authors) in the terms used. Pulmonologists used inappropriate terms to describe pulmonary auscultation as frequently as did nonpulmonologists. In addition, the use of inappropriate terms occurred in all regions of the country. The regions were equivalent in terms of the percentage of inappropriate pulmonary auscultation terms used.

This study does not provide data to explain the causes for the noncompliance with the established standards. The following factors are suggested as being responsible for this noncompliance: insufficient dissemination of the ILSA nomenclature; lack of 
knowledge regarding the advantages of using this new nomenclature; and lack of knowledge regarding the importance, to medical practice, as well as to the teaching process and clinical research, of standardizing the terminology used to describe pulmonary auscultation findings. It should also be borne in mind that, in addition to rales (fine and coarse), there are other abnormal sounds in pulmonary auscultation that originate in the extrathoracic airways (stridor) or in the pleura (friction rub). We found no occurrences of these terms in the articles reviewed in the present study.

Some methodological limitations of this study should be taken into consideration when interpreting the results. The selection of one journal from each of three medical fields was defined a priori as representative of Brazilian medical publications: pulmonology, pediatrics, and clinical practice. Journals from other specialties were not included in this study because they typically present fewer respiratory disease-related articles. The Journal of Pulmonology and the Journal of Pediatrics are indexed journals and are highly representative of their respective fields. Although there are two indexed Brazilian journals in the field of clinical practice, neither is included in the holdings of the library at our university. The Brazilian Journal of Medicine was selected based on the fact the Federal University of Rio Grande Foundation Library hold a nearly complete collection of the issues of this journal. It is questionable as to whether this journal is representative of the clinical practice field. However, the potential bias resulting from our selection could be considered insignificant, since a considerable number of articles published in this journal are related to clinical practice.

In short, the inappropriate use of pulmonary auscultation terms describing adventitious sounds, especially intermittent sounds, remains a common and widespread phenomenon in Brazilian medical publications.

\section{REFERENCES}

1. Pasterkamp H, Kraman SS, Wodicka GR. Respiratory sounds. Advances beyond the stethoscope. Am J Respir Crit Care Med. 1997;156(3 Pt 1):974-87.

2. Renzetti AD Jr. Lung sound terminology. Chest. 1979;76(6):615-16.

3. Garner TK, Duffell GM. Terms for lung sounds. Ann Intern Med. 1979;91(6):928.

4. Wilkins RL, Dexter JR, Smith JR. Survey of adventitious lung sound terminology in case reports. Chest. 1984;85(4):523-5.

5. Donham JA. Rales and rhonchi. Why do we use these terms? Focus Crit Care. 1984;11(5):20-2.

6. Mikami R, Murao M, Cugell DW, Chretien J, Cole P, MeierSydow J, et al. International symposium on lung sounds. Synopsis of preceedings. Chest. 1987;92(2):342-5.

7. Earis J. Lung sounds. Thorax. 1992;47(9):671-72.

8. Piirila P, Sovijarvi AR. Crackles: recording, analysis and clinical significance. Eur Respir J. 1995;8(12):2139-48.

9. Pasterkramp H. The history and physical examination. In: Chernick V, Kening EL, Jr, editors. Disorders of the respiratory tract in children. 5th ed. Philadelphia: W.B. Saunders; 1990. p. 56-8.

10. Sousa RL, Cruz CB, Lima Júnior ZB. Aplicação da semiotécnica pulmonar por médicos residentes e internos de um hospital universitário. Rev Bras Educ Med. 2002;26(1):35-8.

11. Auada MP, Vitória GL, Barros JA. A confusa nomenclatura da ausculta pulmonar brasileira. J Pneumol. 1998;24(3): 129-32. 